\title{
La habilidad negociadora en el Aprendizaje-Servicio con enfoque de la Educación para el Desarrollo: ¿una herramienta pedagógica?
}

Ana Cano-Ramírez ${ }^{\text {a }}$ Francisco Cabrera-Suárez

âUniverdidad de Las Palmas de Gran Canaria, ana.cano@ulpgc.es y ${ }^{\text {b} U n i v e r d i d a d ~ d e ~ L a s ~ P a l m a s ~ d e ~}$ Gran Canaria, fcabrera@dma.ulpgc.es

\begin{abstract}
This paper aims to investigate negotiation as an educational tool within the service-learning experiences implemented as part of the undergraduate degree in social work at the University of Las Palmas de Gran Canaria during the 2012-13-14 and 2016-17-18 academic years. These experiences focus on Education for Development and Global Citizenship (EDGC).

To this end, feedback was obtained from students representing each of the four year groups, once they had completed the task set. Here they provided their views regarding their acquisition of negotiation skills as a result of the experience.

The current study seeks to organize this experience in such a way that it allows for the combined service-learning and EDGC methodology to be explored and to demonstrate the possibilities which this offers for the human development of the students as they experience situations which favour their empowerment, by enhancing their negotiation skills and illustrating their ability to take on responsibilities. This is particularly relevant among social work students, as their professional activity aims to encourage people to undergo processes of this kind in order to overcome the difficulties which have led to their being assisted by the Public Social Services System.
\end{abstract}

Keywords: Service-learning, Education for Development, negotiation skills, critical education theory

\footnotetext{
Resumen

El trabajo que se presenta tiene como objetivo aproximarnos a la negociación como herramienta pedagógica en las experiencias de Aprendizaje-Servicio con enfoque de la Educación para el Desarrollo para la Ciudadanía Global, puestas en práctica durante los cursos 2012-13-14 y 2016-17-18, en Grado de Trabajo Social (Universidad de Las Palmas de Gran Canaria).

Como resultado, una vez finalizada la tarea asumida por los propios estudiantes de los cuatro cursos, hemos podido obtener la valoración que
} 
ellos perciben sobre la adquisición de aprendizaje de la habilidad negociadora a través de la experiencia llevada a cabo.

Se trata aquí de sistematizar la experiencia de manera que ello permita explorar y mostrar el potencial que la metodología ApS, en combinación con la EDCG, aporta en el desarrollo humano de los estudiantes al vivenciar situaciones que contribuyen a su empoderamiento, a través de potenciar las habilidades negociadoras y de verse con capacidad para asumir compromisos, lo que adquiere una relevancia sustancial en los estudiantes de Trabajo Social en tanto que la finalidad de su ejercicio profesional está dirigida a favorecer que las personas afronten procesos de esta índole para superar las dificultades por las que están siendo atendidas en el Sistema Público de Servicios Sociales.

Palabras clave: Aprendizaje-Servicio, Educación para el Desarrollo, habilidad negociadora, teoría crítica educación 


\section{Introducción}

Se presenta en este apartado introductorio elementos que se estiman esenciales para justificar y fundamentar el por qué de la iniciativa pedagógica que se viene impulsando. Por ello, en e aportaremos un marco teórico-conceptual del que se nutre la experiencia, así como de la presentación grosso modo sobre los fundamentos que construye el currículum institucional-profesional y el currículum institucional-académico.

En los apartados siguientes presentamos los objetivos perseguidos, la experiencia de innovación educativa que impulsamos, y de la que hemos ido recopilando una serie de resultados a tenor de los objetivos y procedimiento aplicado. De todo ello, alcanzamos unas conclusiones finales que reportamos, para finalizar con las fuentes bibliográficas con las que hemos tratado de ahondar en el conocimiento del contexto y de la acción docente.

Antes de continuar, estimamos pertinente advertir al lector que, el trabajo que aquí nos ocupa es, amén de la estructura convencional que se evoca en este tipo de documento, fruto de una construcción combinada de la teoría y la praxis. En este sentido, recomendamos considerar que los contenidos referidos al currículum institucional-profesional y currículum institucional-académico son fruto y, por tanto, también se constituyen como resultados, de la observación a la que la práctica docente nos ha sumergido, permitiéndonos analizar y reflexionar sobre el sentido y la coherencia de la misma respecto a aquellos. Procede decir que, de alguna forma, lo que aquí estamos tratando es de sistematizar una experiencia de Aprendizaje-Servicio haciendo un "servicio docente" mediante el que, a su vez, estamos aprendiendo como profesores.

\section{Fundamentación de la habilidad negociadora en la experiencia docente}

En esta sección vamos a realizar un recorrido por la literatura que nos aporta elementos que justifican la necesidad de trabajar con nuestros estudiantes la habilidad negociadora. Para ello acudimos, en primer lugar, al marco conceptual del enfoque metodológico de la Educación para el Desarrollo para la Ciudadanía Global (en adelante EDCG) y del instrumento práctico que lo hace posible, el Aprendizaje-Servicio (en adelante ApS).

En segundo lugar, nos adentramos en el currículum institucional-profesional, para desvelar qué se nos dice desde el currículum profesional al respecto. En tercer y último lugar, dedicamos un espacio para justificar la experiencia docente desde el enfoque de la narrativa académica.

\subsection{Fundamentación teórica}

A continuación se aportan elementos de fundamentación conceptual con el propósito de delimitar el trabajo pedagógico objeto de atención del presente documento. En esta ocasión adoptamos la definición de ApS como "una forma de educación basada en la experiencia, en la que el aprendizaje se produce a través de un ciclo de acción y reflexión gracias al cual los estudiantes trabajan con otros compañeros en un proceso de aplicación de lo que han aprendido a los problemas de la comunidad y, al mismo tiempo, reflexionan sobre la experiencia de perseguir objetivos reales para la comunidad e incrementar su propia

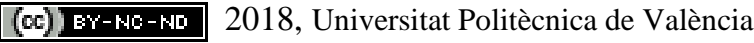

Congreso In-Red (2018) 
comprensión y destrezas, es decir, desarrollan de manera conexa las múltiples dimensiones humanas y cultivan la responsabilidad cívica y social” (Eyler \& Gilers, 1999, citado en Francisco y Moliner, 2013:71). A este ejercicio profesional reflexionado, añadimos la perspectiva de Aramburuzabala (2013:6) cuando señala un ApS guiado por la justicia social, con especial referencia a los colectivos en situación de vulnerabilidad social, todos ellos aspectos trascendentales de los trabajadores sociales.

Esta definición sostiene exquisitos hilos de conexión con lo que viene a definirse como EDCG, al entenderse ésta como "un proceso educativo, continuo, democrático, emancipador, basado en valores humanísticos, para que la persona tome conciencia crítica frente a las complejas relaciones sociales, económicas y políticas, y se posicione con sensibilidad contra las injusticias sociales y actúe para transformarlas, a la vez que construir un mundo más justo para ser feliz” (Cano, 2014:421).

Consideradas las definiciones ApS y de la EDCG, se desprende que sostienen una íntima relación en tanto que una se constituye en oportunidad pedagógica de la otra. Dicho esto, este trabajo pretende evidenciar en qué medida, el ApS contribuye a aprendizajes desde el enfoque de la EDCG, en concreto identificando la adquisición de la capacidad de negociar y llegar a compromisos, como habilidad que se puede abordar en el marco del currículum oculto, es decir, aprendizajes que adquieren los estudiantes sin que éstos sean objeto intencionado ni consciente del currículum institucional ni del currículum ofrecido (Zabalza, 2003: 32-33; Alvárez Méndez, 2009: 208; Acaso, 2012: 45).

Siguiendo con la EDCG, cabe decir que se nutre de las teorías constructivistas de la psicología y de la las teorías contemporáneas de la pedagogía. Entre ellas, se desprende con especial protagonismo las siguientes: Pedagogía de la Liberación de Paulo Freire, la Construcción Histórico-socio-cultural del Conocimiento de Vigotsky, la Teoría del Aprendizaje Significativo de Ausubel y la Teoría de los Intereses del Conocimiento de Habermas (Díaz, 2010), teorías, todas ellas, inspiradoras de la iniciativa pedagógica que nos ocupa.

Sin pretención de entrar a explicar las teóricas citadas, sí resulta de interés hacer presente, por el objeto que nos ocupa, el planteamiento que Habermas sostiene cuando apunta a que la educación persigue alguno de los tres intereses que él identifica como: interés práctico, interés técnico e interés emancipatorio. Para él, la ciencia se ordena según estos tres tipos de intereses, y el saber se adquiere de las siguientes formas que se corresponde con cada uno de los intereses señalados: empírico-analítico, histórica-hermenéutico y crítico (Grundy, 1998). Atendiendo a ello, y considerando las palabras de Boni (2005), cuando señala que la finalidad de la EDCG es "contribuir a desarrollar unos conocimientos, habilidades, valores y actitudes coherentes con el objetivo político que propone: la consecución del desarrollo humano sostenible para todo el planeta”, a partir de una educación crítica y emancipadora, resulta que la Teoría de Habermas que refiere al interés emancipador es la que mantiene coherencia con la EDCG, en el que nos posicionamos como docentes.

A este respecto, y siguiendo con la propuesta de Habermas, Grundy (1998) desarrolla de manera muy interesante la perspectiva del currículum, que debe contemplar una finalidad emancipadora, para que los estudiantes adquieran autonomía y pensamiento crítico. Entre

(c) EY-NC-ND 2018, Universitat Politècnica de València 
estas sugestivas ideas aparece el concepto de "currículum negociado" como modelo pedagógico que evidencia el compromiso activo de los profesores con sus alumnos. Este currículum negociado resulta de un acto de negociación entre ambas partes, no de forma espontánea ni improvisada, sino como resultado de un ejercicio de reflexión sistemático. En este sentido, las experiencias docentes que abordamos persigue alcanzar ese currículum negociado que plantea Grundy.

Añade el autor que el aprendizaje se basa en un proceso de investigación-acción, de manera que a la vez que hace, indaga e investiga sobre ello, reflexiona, analiza y actúa, mejorando la praxis, para volver a ella. Esta es la práctica pedagógica que puede garantizar el proceso emancipador, a partir de desarrollar esa actitud crítica. Este interés emancipador requiere que la acción vaya acompañada necesariamente de la reflexión (Grundy, 1998). Este proceso de investigación-acción nos está permitiendo desvelar el alcance de los objetivos de empoderamiento de los estudiantes, a través de la revisión continuada de la praxis guiada por la teoría, retroalimentándonos continuamente.

Apunta también la teoría de los intereses de la educación, en concreto siguiendo con la emancipadora, que cuando se evalúa a un estudiante, se ha de hacer desde un posicionamiento crítico, no solo sobre los resultados obtenidos del aprendizaje, sino sobre cómo se han producido aquellos. Para la realización de esta evaluación, solo tiene poder reconocido los que han experimentado el proceso, por lo que queda excluida la intervención de cualquier agente externo que tenga interés por evaluar (Grundy, 1998).

De todo ello se puede extraer la idea de que la acción evaluadora del profesor sea cuestionada, en tanto que este no conoce con exactitud qué ha experimentado y qué cambios se han producido en sus estudiantes. Si lo que se quiere es estimular un aprendizaje orientado al desarrollo de habilidades o competencias superiores (pensamiento crítico y creativo, capacidad de resolución de problemas, capacidad de análisis y síntesis, etc.), es necesario practicar una evaluación que vaya en consonancia con ello (Álvarez Méndez, 2009). Este aprendizaje sitúa al estudiante como el principal agente para identificar y reconocer sus propios aprendizajes, quedando vinculado al modelo de autoevaluación, como la fórmula más coherente con este modelo de aprendizaje. Este criterio ha sido perfilado en la segunda etapa de la experiencia pedagógica que presentamos, estableciendo los propios estudiantes cúales eran los criterios de evaluciación del trabajo grupal realizado, así como el modelo de evaluación a aplicar.

Así mismo, compartimos con De Paz (2009) que es necesario considerar el compromiso que los estudiantes adoptan con las formas de trabajar. Este compromiso está íntimamente relacionado con su participación en tomar decisiones sobre lo que ha de hacer, decisiones que resultan de los procesos de negociación, especialmente cuando la decisión a adoptar incluye planos de carácter grupal/colectivo, como es el caso que intentamos proyectar.

A ello se suma que en la propuesta de trabajo pedagógico coincidimos con Bain (2005: 45) cuando esboza que la práctica docente desde la EDCG necesita "ajustar los objetivos y el nivel conceptual a la situación de los estudiantes”. Esta indicación exige identificar qué conocimientos de partida tienen los estudiantes para definir los resultados que se espera deben alcanzar los mismos. Ello requiere que las dificultades del tema deben presentarse según el nivel de comprensión del grupo objetivo, pero debe tener una dificultad algo 
mayor de lo que ya sabe, a fin de que se vaya incrementando sus conocimientos. No obstante, se debe cuidar que no sea de tal complejidad que le impida asimilarla. Se tiene que considerar que el estudiante no llega aséptico al aula, sino que viene con una cubierta de su contexto social y cultural que ha de considerarse tanto desde las ventajas como desde las desventajas que conlleve (Gimeno, 2005). Por todo ello, la apertura de espacios y tiempos de debate compartidos en el aula, ha favorecido que tanto los estudiantes como la docente tomemos pulso a cómo nos vemos a nosotros mismos ante la innovación docente que afrontamos, en tanto que exigirá sacar a la luz, además de nociones teóricas, habilidades y actitudes que en el modelo docente tradicional se encuentran cercenados.

Para finalizar esta sección, como apuntan Juanjo Celorio (1995, citado en Argibay y Celorio, 2005) y Luque (2011), los contenidos deben sostener una lógica en función a problemas relevantes, que conecten con los intereses de los estudiantes. La propuesta que formulamos expone al estudiante a la búsqueda de organizaciones que gestionan servicios sociales, atendiendo a sus inquietudes, intereses o preocupaciones. Con ello, nos sumamos a la estrategia que De Paz (2009) propone respecto a establecer iniciativas que integran a los recursos de la comunidad y del entorno para educar en problemáticas de relevancia académica personal y de relevancia social, vinculándose con el aprendizaje de la teoría de Vigotsky, que se sostiene en la construcción histórica-social y cultural. En este sentido, los intercambios constantes con otras personas portadoras de creencias, de diferentes formas de vida cotidiana, así como de la propia formación permanente como facilitadores de los profesores, enriquecen y contribuyen a la construcción de nuevos significados del mundo (Hegoa/Incyte, 2003: 59). "Todo ello sostiene coherencia con que la pedagogía debe corresponder a las raíces culturales y al contexto comunitario y político”, esto es a las condiciones y características del grupo (Hegoa/Incyte, 2003), a la vez que con el aprendizaje significativo, de la Teoría de Ausubel.

\subsection{Fundamentación con base al currículum institucional-profesional}

A partir de 2014, y como resultado de un largo periodo de revisión, el Comité Ejecutivo de la Federación internacional de Trabajadores Sociales y la Junta de la Asociación Internacional de Escuelas de Trabajo Social, alcanzan una definición global consensuada de Trabajo Social: "el Trabajo Social es una profesión basada en la práctica y una disciplina académica que promueve el cambio y el desarrollo social, la cohesión social, y el fortalecimiento y la liberación de las personas. Los principios de la justicia social, los derechos humanos, la responsabilidad colectiva y el respeto a la diversidad son fundamentales para el Trabajo Social. Respaldada por las teorías del Trabajo Social, las ciencias sociales, las humanidades y los conocimientos indígenas, el Trabajo Social involucra a las personas y las estructuras para hacer frente a desafíos de la vida y aumentar el bienestar."

Dicho esto, resulta evidente la complejidad del ejercicio profesional de los trabajadores sociales, por lo que su transcurso por la universidad ha de contribuir de manera clara e intencionada a la capacitación de habilidades y actitudes que le permita poner en práctica las nociones conceptuales que configuran la profesión. Ello exige una profunda reflexión sobre el modelo educativo dominante definido en los planes de estudio y las prácticas

(cc) EY-NC-ND 2018, Universitat Politècnica de València

Congreso IN-RED (2018) 
metodológicas docentes que se están llevando a cabo en las aulas, y buscar respuesta a si se está haciendo una formación que atiende a las exigencias de la sociedad, entendida como el sector de población privadas de derechos sociales y que se encuentra demandando respuestas a su situación de vulnerabilidad y/o exclusión social, de una parte, pero también del conjunto de la ciudadanía privadas de procesos de desarrollo comunitario y participación social que les permita alcanzar un nivel de bienestar social que dignifique sus vidas.

\subsection{Fundamentación según currículum institucional-académico}

Ahora vamos a centrar la atención sobre el por qué la negociación se constituye en un necesario foco de atención como habilidad objeto de aprendizaje, la cual se proyecta desde la práctica pedagógica en la titulación de Grado de Trabajo Social.

\subsubsection{Libro Blanco de Grado de Trabajo Social}

Con relación a la capacidad negociadora de la que han de dotarse los trabajadores sociales, el Libro Blanco de Grado de Trabajo Social nos indica que “(...) la competencia laboral implica movilizar una serie de atributos para trabajar exitosamente en diferentes contextos y bajo diferentes situaciones emergentes. Los conocimientos se combinan con las habilidades y la percepción ética de los resultados del trabajo en el intorno y el entorno, con la capacidad de comunicarse y entender los puntos de vista de los compañeros de profesión y usuarios/clientes, la habilidad para negociar e intercambiar informaciones, etc.” (Agencia Nacional de Evaluación de la Calidad y Acreditación, 2004:110).

Continúa el Libro Blanco señalando cinco ámbitos competenciales profesionales, identificando los siguientes criterios de realización que requieren de la habilidad negociadora de los profesionales:

- Mediar y negociar cuando las personas tienen puntos de vista diferentes.

- Negociar cuándo los planes deben ser ampliados o reducidos y dónde se necesita y está disponible el apoyo adicional necesario.

- Trabajar con el sistema cliente identificando, negociando y seleccionando la mejor forma de representación.

- Realizar el seguimiento del nivel de riesgo y renegociando la acción planificada cuando se producen cambios.

- Negociar y establecer las propias responsabilidades profesionales dentro de la relación.

- Negociar y establecer las propias expectativas con respecto a la relación, y las que otros tienen del trabajador social dentro de la relación.

1.3.2. Memoria de verificación del Título de Grado de Trabajo Social de la ULPGC 20102011

La Universidad de Las Palmas de Gran Canaria (ULPGC), con la llegada del EEES, y con todo el engranaje de ordenamiento de sus títulos de grado (Ley Orgánica 4/2007, de 12 de 
abril, por la que se modifica la Ley Orgánica 6/2001, de 21 de diciembre, de Universidades, y Real Decreto 1393/2007, de 29 de octubre, por el que se establece la ordenación de las enseñanzas universitarias oficiales), define en la memoria de verificación del Título de Grado de Trabajo Social de la ULPGC 2010-2011, lo siguiente con relación a la habilidad negociadora que ha de ser abordada durante el proceso formativo de los futuros trabajadores sociales.

De un lado, entre los resultados de aprendizajes que ha de alcanzar los estudiantes en la materia relativa a Métodos, Modelos y Técnicas del Trabajo Social, se tiene el hecho de que: sea capaz de consultar y cooperar con otros, incluidos los usuarios de servicios, negociando los límites institucionales y profesionales y reconociendo las diferencias de identidad o lenguaje; sea capaz de diseñar, implementar y evaluar planes de intervención con las personas atendidas y otros profesionales negociando el uso de servicios y revisar la eficacia de dichos planes; y sea capaz de aplicar las estrategias de resolución de conflictos por medio de la negociación y la mediación

De otro lado, como resultados de aprendizaje en la materia relativa a Habilidades Sociales y de Comunicación del Trabajo Social, el estudiante ha de ser capaz de establecer relaciones profesionales al objeto de identificar la forma más adecuada de intervención y de aclarar y negociar la finalidad de tales contactos y los limites de su implicación.

Y, finalmente, con relación al Trabajo Fin de Grado, se persigue que se alcance como resultado de aprendizaje que sea capaz de diseñar, implementar y evaluar proyectos de intervención social y planear de forma negociada una secuencia de acciones y seguir y evaluar su desarrollo.

\section{Objetivos}

La finalidad del trabajo es demostrar si la puesta en práctica de metodologías docentes basadas en el ApS, permiten el aprendizaje de las habilidades que define a la EDCG.

En este trabajo hemos focalizado la atención en el objetivo de analizar si la aplicación del ApS favorece, al margen de las competencias explícitas y específicas de la asignatura que se han indicado en las guías docentes, la adquisición de la "capacidad de negociar y de llegar a acuerdos”, habilidad definida por la EDCG (Boni, et. al., 2013).

La hipótesis de trabajo es que el ApS es una metodología docente que contribuye a la adquisición de los aprendizajes esta habilidad.

El proceso del trabajo de indagación ha consistido en la elaboración de un cuestionario, recogida de datos, transcripción de la información al programa excel, presentación de resultados, análisis de los datos obtenidos y elaboración de conclusiones.

El instrumento de recogida de datos es la encuesta, con tipo de pregunta cerrada (¿cuál de los siguientes contenidos se adquiere con la experiencia Aps?), con opción de respuesta dicotónica ("sís" o "no").

La recogida de datos se efectúa el último día de clase, a la totalidad de estudiantes que asisten al aula. En el momento de cumplimentar la encuesta, los estudiantes desconocen la

(c) BY-NC-ND 2018, Universitat Politècnica de València

Congreso IN-RED (2018) 
existencia de los atributos, pues no se configuran de manera intencionada en la guía docente, ni se ha explicitado verbalmente durante el semestre, lo que significa que responden ipso facto su valoración sobre el aprendizaje/adquisición que han obtenido sobre aquellos.

\section{Desarrollo de la innovación}

La innovación docente que hemos llevado a cabo se circunscribe a la puesta en práctica de la herramienta del ApS, con enfoque de EDCG. Hemos tenido dos modelos de experiencias: uno durante los cursos 2012-13 y 2013-14 (con estudiantes de primer curso, asignatura Organización de los Servicios Sociales, y de segundo curso con la asignatura Programas y Prestaciones de los Servicios Sociales), y un segundo modelo durante los cursos 2016-17 y 2017-18 (con estudiantes de segundo curso con la asignatura Programas y Prestaciones de los Servicios Sociales).

Como rasgos comunes a ambos episodios se destaca:

- El carácter voluntario de los estudiantes para optar a llevar a cabo la experiencia, en ningún momento impuesta por la docente. Este formato favorece de manera esencial contar con el compromiso de los estudiantes con la acción pedagógica perseguida.

- Se viven situaciones de debate y reflexión compartidas en el aula, a la vez que a nivel intragrupal, e individual. Se trata de espacios de negociación y toma de decisiones al interno del aula.

- En todos los casos, los estudiantes, bien a nivel individual (primera experiencia), o grupal (segunda) asumen dirigirse directamente a organizaciones gestoras de servicios sociales con las que deberán negociar y acordar la posibilidad de llevar a cabo la actividad de ApS y/o el trabajo grupal. No se produce en ningún momento la intermediación por parte de la docente entre estudiantes y organizaciones. Se trata de negociación y toma de decisiones al externo del aula.

Estos tres elementos sitúan al estudiantado en conexión con lo académico y con la realidad del contexto, desempeñando un papel de relaciones complejas que repercuten en sus aprendizajes.

Los asuntos sometidos a negociación y a toma de decisiones que conlleva a compromisos suponen dos fases claves: la primera fase versa sobre ¿asumir (o no) un modelo de aprendizaje dirigido/ cerrado/ no negociado/ impuesto por la docente? ¿asumir (o no) el reto de intentar aprender de otra manera más activa, participativa y en contacto con el entorno y la realidad?. En todas las experiencias este periodo de debate, análisis y reflexión se torna como mecanismo pedagógico de aprendizaje, exigiendo la puesta en práctica de habilidades $\mathrm{y}$ actitudes individuales en el aula.

Respecto a esta primera fase, en la primera etapa (2012-14), la propuesta acordada se caracterizó por una toma de decisión individual por la que el estudiante acogido a esta modalidad debía contactar, explicar, negociar y tomar decisiones sobre el compromiso que asumía directamente con una organización (escogida por el mismo) gestora de programas o prestación de servicios sociales. En esta ocasión se trata de sustituir el examen como 
estrategia de evaluación por la experiencia de ApS que conlleva la contribución del estudiantes de 15 h (primer año) y 20 h, (en el seguno) de presencia y participación en las actividades que la organización y estudiante acordaron, atendiendo a las necesidades de la misma y en consonancia con contenidos de la asignatura, de manera que cada estudiante ha de definir, desde el inicio, de manera consciente e intencionada, qué objetivos de aprendizaje y qué objetivos de servicio se propone (con relación a esta experiencia puede ampliarse información en: Cano, Díaz y Guedes, 2013; Cano y Cabrera, 2015).

En la segunda etapa (2016-18), la decisión inicial adoptada por los grupos de trabajo de la asignatura, afectaría al grupo clase en su conjunto, de manera que la decisión última supondría que todos trabajaríamos el mismo proyecto. En esta ocasión, la propuesta se circunscribe al trabajo grupal obligatorio establecido en la guía docente. Tras el acuerdo adoptado, toda la clase trabajó en torno a la organización y celebración de lo que fueron los II y III Encuentros de Sensibilización sobre las Realidades Sociales. Durante el proceso, estudiantes y docente abordamos conjuntamente la idealización, coordinación, organización, y celebración de sendos encuentros. Cada una de estas estrategias de trabajo conllevó reflexión, negociación y toma de decisiones, que suponen compromisos de realización de tareas y acciones dirigidas al objetivo de celebración del encuentro, aprendizaje por proyecto que, pedagógicamente, se torna como mecanismo de "disculpa" sobre el que gira todo el proceso. Adoptamos decisiones sobre: qué hacer, cómo hacerlo, cuándo, con qué, con quiénes y dónde hacerlo (el primer año se hizo en las instalaciones de la Facultad de Ciencias Jurídicas y la Facultad de Economía, Empresa y Turismo; el segundo año se celebró en la Escuela de Ingenierías Industriales y Civiles y en la Escuela de Ingeniería, Telecomunicaciones y Electrónica). En un momento avanzado del proceso de trabajo, se planea la idealización de criterios de evaluación y modelo de evaluación (autoevaluación y coevaluación) (con relación a esta experiencia puede ampliarse información en: Cano: 2015; Cano y Cabrera: 2016, 2017a, 2017b y 2017c).

\section{Resultados}

Atendiendo a la finalidad definida anteriormente, presentamos a continuación los resultados obtenidos. Estos refieren a la valoración que los estudiantes perciben respecto a si la experiencia pedagógica que han desarrollado, les ha permitido (o no) mejorar su "capacidad de negociar y llegar a compromisos”.

En esta ocasión nos interesa obtener una visión global y comparada entre las dos experiencias (2012-14 y 2016-18) por lo que centraremos la atención en el análisis de los datos obtenidos de ambas.

Se tiene que, de un total de 246 encuestas (100\%), 197 estudiantes (80,1\%) responden positivamente respecto a haber desarrollado más esta habilidad a través de la experiencia de ApS que se ha llevado a cabo en su asignatura.

De ese total, podemos distinguir que durante la experiencia de los cursos 2012-13-14 casi las tres cuartas partes de las respuestas obtenidas responden afirmativamente $(72,1 \%)$, mientras que este dato queda superado por la experiencia de los cursos 2016-17-18, con un $88,9 \%$. 
Tabla 2. Resultados del análisis factorial

\begin{tabular}{cccc}
\hline $\begin{array}{c}\text { Experiencias } \\
\text { cursos }\end{array}$ & Total encuestas & $\begin{array}{c}\text { Total respuestas positivas: } \\
\text { adquisición de la capacidad de } \\
\text { negociar y de llegar a compromisos }\end{array}$ & Porcentaje \\
\hline $2012-13-14$ & 129 & 93 & 72,1 \\
$2016-17-18$ & 117 & 104 & 88,9 \\
Totales & 246 & 197 & 80,1 \\
\hline
\end{tabular}

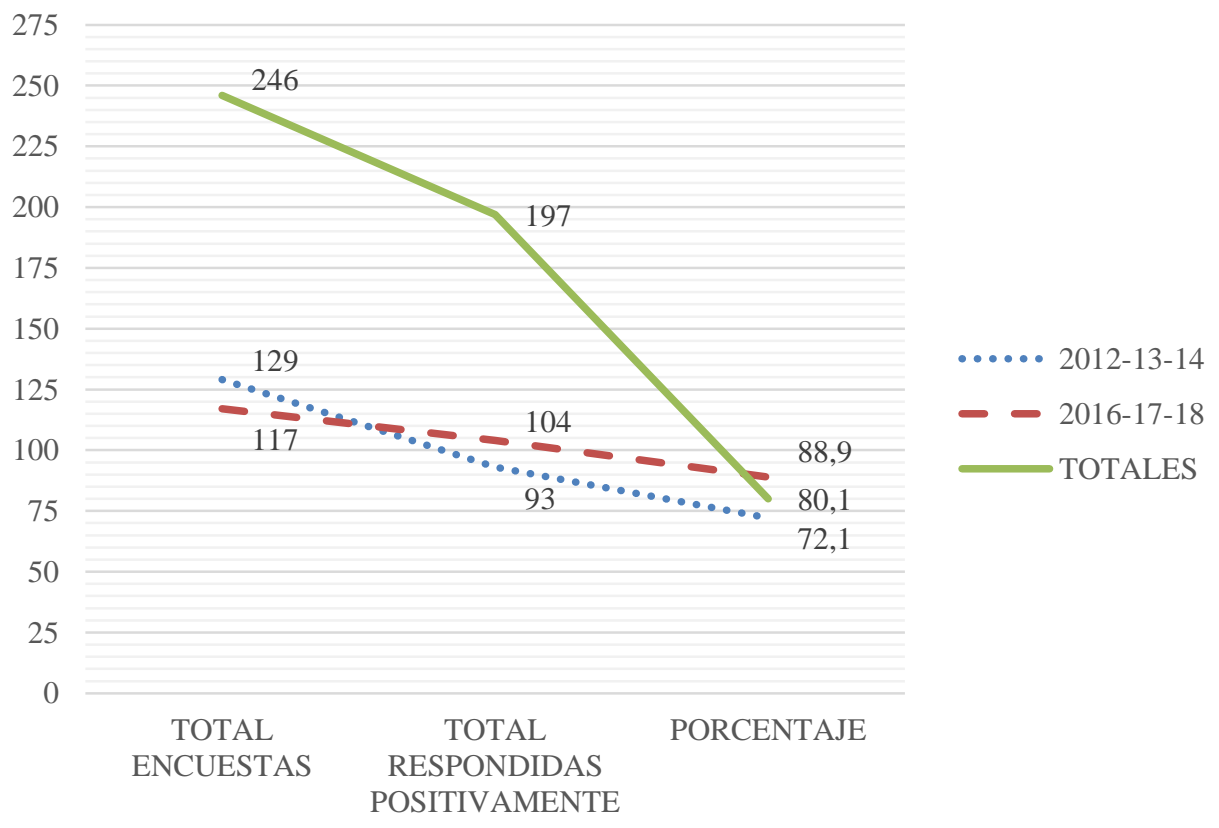

Gráfica 1 Resultados de la encuesta

\section{Conclusiones}

En primer lugar, y atendiendo a la narrativa observada en el apartado de fundamentación de la propuesta docente que presentamos, podemos concluir que la "capacidad de negociar y llegar a compromisos" se configura como una de las destrezas contempladas en el currículum profesional y el currículum académico, sosteniendo una coherencia y sintonía entre sí, a la par que dotando de sentido a la práctica docente que estamos aplicando.

Centrándonos ahora en las experiencias, dadas las diferencias de procedimiento entre los dos formatos realizados (2012-14 y 2016-18), y atendiendo a las respuestas obtenidas, se concluye que el modelo seguido en los dos últimos años, acentúa su contribución a la 
adquisición de esta habilidad. Este positivo progreso y sistematización de la praxis, contribuye a la maduración y consolidación del enfoque pedagógico de la EDCG y enfoque metodológico con el ApS.

Además, se confirma que, por un lado, sin estar explícita esta competencia en las guías docentes de la asignaturas (y sin posibilidad de ello en tanto que no están reconocidas dentro de las materias del Plan de Estudios del Titulo, esto es el currículum institucional) y, por otro lado, cumpliendo con los objetivos y aprendizajes previstos en la guía docente de la asignatura (currículum ofrecido), el enfoque metodológico aplicado permite adquirir otros aprendizajes, configurándose como currículum oculto y que, en este caso, complementa al currículum real, como aprendizajes que efectivamente adquieren los estudiantes.

Por tanto, podemos afirmar que la hipótesis de indagación se confirma, en tanto que el ApS, con enfoque de EDCG favorece la adquisición de la capacidad negociadora y de llegar a compromisos.

Poniendo la mirada hacia el futuro, y atendiendo a todo lo anterior, visto los datos arrojados, se propone que durante la revisión del plan de estudios del Grado de Trabajo Social se estime la conveniencia de incorporar la adquisición de la competencia en las asignaturas de la materia de servicios sociales, permitiendo visibilizarlo en el currículum ofrecido, esto es, en la guía docente.

Y, para finalizar, esta introspección que venimos haciendo, nos insta a profundizar respecto la identificación, aanálisis y reflexión sobre los hitos que caracterizan el procedimiento pedagógico que están contribuyendo al desarrollo de la habilidad, de manera que nos permita introducirlas mejoras en futuros cursos.

\section{Referencias}

ACASO, M. (2012). Pedagogías invisibles. El espacio del aula como discurso. Madrid: Catarata.

AGENCIA NACIONAL DE EVALUACIÓN DE LA CALIDAD Y ACREDITACIÓN (2004). Libro Blanco Título de Grado de Trabajo Social.

$<$ http://www.aneca.es/var/media/150376/libroblanco_trbjsocial_def.pdf> [Consulta: 10 de abril de 2017].

ÁLVAREZ MÉNDEZ, J.M. (2009). “Evaluar el aprendizaje en una enseñanza centrada en las competencias”. Gimeno Sacristán, J. (Comp) en: Educar por competencias ¿qué hay denuevo?. Madrid: Ediciones Morata. Pp 206-234.

ARAMBURUZABALA, P. (2013). “Aprendizaje-Servicio: Una herramienta para educar desde y para la justicia social” en Revista internacional de educación para la justicia social, vol. 2, Núm. 2, p. 5-11. <http://www.rinace.net/riejs/numeros/vol2-num2/editorial.pdf> [Consulta: 7 de abril de 2017].

ARGIBAY, M. Y CELORIO, G. (2005). Educación para el Desarrollo. Vitoria: Servicio Central de Publicaciones del Gobierno Vasco-HEGOA.

BAIN (2005). Lo que hacen los mejores profesores de universidad. Valencia: Servicio de Publicaciones de la Universidad de Valencia.

(cc) EY-NC-ND 2018, Universitat Politècnica de València

Congreso IN-RED (2018) 
BONI, A. (2005). Tesis La Educación para el Desarrollo en la enseñanza universitaria como una estrategia de la cooperación orientada al desarrollo humano. Universidad de Valencia. <http://ccd.webs.upv.es/files/Fondo_documental/Tesis/Alejandra\%20Boni Aristizábal.pdf>

BONI, A., LÓPEZ, E. Y R. BARAHONA (2013). “Approaching quality of global education practices through action research. A non-governmental development organization-university collaborative experience”en International Journal of Development Education and Global Learning 5(2), pp. $31-46$.

CANO-RAMÍREZ, A., DÍAZ GONZÁLEZ, N. Y VIERA GUEDES, D. (2013). “AprendizajeServicio: una práctica docente que acerca a los estudiantes de Grado de Trabajo Social a la realidad social y profesional”. Universidad de Málaga. XII Congreso Estatal de Trabajo Social: la intervención social en tiempos de malestares. Madrid: Consejo General de Trabajo Social.

CANO-RAMÍREZ, A. (2014). Exploración de las prácticas docentes con enfoque de Educación para el Desarrollo para la Ciudadanía Global. Aproximación diagnóstica en los títulos de grado de las universidades españolas tras la implementación del EEES. Tesis Doctoral. Las Palmas de Gran Canaria: Universidad de Las Palmas de Gran Canaria. <http://hdl.handle.net/10553/12352> [Consulta: 21 de marzo de 2017]

CANO-RAMÍREZ, A. (2015). Aprendizaje-Servicio y Educación para el Desarrollo: aproximación a los aprendizajes a partir de la experiencia docente. Foro de Investigación en Educación para el Desarrollo y Ciudadanía Global. Universidade de Coruña.

CANO-RAMÍREZ, A. Y CABRERA SUÁREZ, F. (2016). “Estudio sobre la adquisición de habilidades desde la perspectiva de la Educación para el Desarrollo a partir de la experiencia de Aprendizaje-Servcio en Grado de Trabajo Social de la ULPGC” en González Barea, E.M. y Moreno Yus, MA. (Eds). Aprendizaje-Servicio, desarrollo y comunidades profesionales de aprendizaje en Educación Superior. Murcia: Universidad de Murcia. Vol. II, pp 274-279.

CANO-RAMÍREZ, A. Y CABRERA-SUÁREZ, F. (2017a). ApS y procesos de empoderamiento de los estudiantes a través de la organización de un evento: II Encuentro de Semnsibilización sobre Realidades Sociales en la ULPGC. VIII Congreso Nacional y III Internacional de AprendizajeServicio Universitario ApS-U8. Sevilla: Universidad Pablo Olavide. Pendiente de publicación.

CANO-RAMÍREZ, A. Y CABRERA SUÁREZ, F. (2017b). "Estudio sobre la adquisición de habilidades de la Educación para el Desarallo como resultado de la experiencia docente de aprendizaje-servicio”. Universidad Jaume I. VI Jornada Nacional sobre Estudios Universitarios. Castellón.

CANO-RAMÍREZ, A. Y CABRERA-SUÁREZ, F. (2017c). “Motivaciones de los estudiantes pada acogerse a una metodología docente basada en el Aprendizaje-Servicio” en J. Carrillo-Rosúa, J.L. Arco-Tirado \& F.D. Fernández-Martín (eds.). Investigando la mejora de la enseñanza universitaria a través del aprendizaje-servicio (pp. 61-67). Granada: Editorial Universidad de Granada. <http://hdl.handle.net/10481/46881> [Consulta: 27 de enero de 2018]

DE PAZ ABRIL, D. (2009). "La escuela como espacio de diálogo. Un camino de esperanza”, en Consorcio Conectando Mundos, Educar para una ciudadanía global. Construir un mundo justo desde la escuela. España: Intermón Oxfam. Pp.95-99.

DÍAZ PINTO, C.F. (2010). Viejas y Nuevas Ideas en Educación. Una historia de la pedagogía. Madrid: Editorial Popular.

FEDERACIÓN INTERNACIONAL DE TRABAJADORES SOCIALES (2014). "La agenda global para el trabajo social $y$ el reporte del progreso de desarrollo social”. <http://cdn.ifsw.org/assets/ifsw_105524-8.pdf> [Consulta: 24 de febrero de 2017] 
FRANCISCO, A. Y MOLINER, L. (2010). "El Aprendizaje Servicio en la Universidad: una estrategia en la formación de ciudadanía crítica” en Revista Interuniversitaria de Formación del Profesorado, 13(4). <http://www.aufop.com> [Consulta: 2 de octubre de 2016]

GIMENO SACRISTÁN, J. (2005). La educación que aún es posible. Madrid: Ediciones Morata.

GRUNDY, S. (1998). Producto o praxis del currículum. $3^{\mathrm{a}}$ edición. Madrid: Ediciones Morata.

LEY ORGÁNICA 4/2007, de 12 de abril, por la que se modifica la Ley Orgánica 6/2001, de 21 de diciembre, de Universidades (BOE núm. 89, del 13 de abril de 2007). <http://www.boe.es/boe/dias/2007/04/13/pdfs/A16241-16260.pdf>

POLYGONE (2003). Mosaico educativo para salir del laberinto. Red Internacional de educación para el desarrollo y educación popular. Vitoria-Gasteiz: HEGOA/Incyte.

REAL DECRETO 1393/2007, de 29 de octubre, por el que se establece la ordenación de las enseñanzas universitarias oficiales (BOE núm. 260, del 30 de octubre de 2007). $<$ https://www.boe.es/buscar/act.php?id=BOE-A-2007-18770> [Consulta: 13 de enero de 2017]

Universidad de Las Palmas de Gran Canaria. Proyecto de Título de Grado de Trabajo Social de la Universidad de Las Palmas de Gran Canaria 20110-11. <www.ulpgc.es> [Consulta: 13 de enero de 2017]

ZABALZA, M.A. (2003). Competencias docentes del profesorado universitario. Calidad y desarrollo profesional. Madrid: Narcea.

(cc) EY-NC-ND 2018, Universitat Politècnica de València 